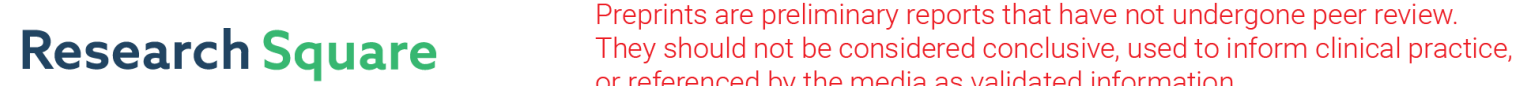 \\ or referenced by the media as validated information. \\ Theoretical Proof that Entangled Polarized Photons Allow Superluminal Communication
}

Vinicius Ritzmann ( $\square$ ritzmann.vinicius@gmail.com ) https://orcid.org/0000-0002-9145-5769

\section{Research Article}

Keywords: Quantum Optics, No-Signalling Theorem, Quantum Entanglement

Posted Date: April 12th, 2021

DOI: https://doi.org/10.21203/rs.3.rs-408717/v1

License: (a) (i) This work is licensed under a Creative Commons Attribution 4.0 International License. Read Full License 


\title{
Theoretical Proof that Entangled Polarized Photons Allow Superluminal Communication
}

\author{
Ritzmann, V. A.* \\ Independent Scholar \\ (Dated: April 10, 2021)
}

\begin{abstract}
In Quantum Mechanics, two particles are entangled if their physical states depend on one another's so that if we find one of them in state A, for example, we will be sure that the other is in state B. However, until the state of a quantum particle is measured, it will be in a superposition of states, being in neither one nor the other until then, so when an entangled particle is measured, its pair also assumes a state instantly and regardless of how far away it is from the other particle at that time. As promising as it could be to use this fact for instantaneous communication, Quantum Mechanics seems to claim this is impossible, as no method ever invented to do this has worked until today. What we demonstrate here theoretically is that with a protocol and simple optical devices, two people who share polarized entangled pairs of photons can send information to each other faster than light. If this model of communication proves to be experimentally functional, we will have a contradiction to Einstein's theories of relativity, and otherwise, we will have Quantum Mechanics predicting something that does not happen in real life. This result, therefore, shows there is something fundamental about the universe we do not know yet. One of these theories must be mistaken and both deal with fundamental aspects of reality, such as the dynamics of space and time, and the particles that almost all matter around us are made of. Besides, this result is of great relevance also because it has immediate applications in several areas if the model works experimentally, as in space exploration and security, since it will allow the creation of noninterceptable instantaneous communication technologies.
\end{abstract}

\section{INTRODUCTION}

The possibility of superluminal communication with quantum entanglement has already been the subject of a great debate in Quantum Mechanics. This debate became evident after the publication of the article by Einstein, Podolsky, and Rosen in 1935 [1], which questioned whether Quantum Mechanics could be considered complete as it allowed particles to assume the so-called entangled state, as is discussed in the article, this state seemed to allow superluminal communication, what is impossible according to the Theories of Relativity.

In Quantum Mechanics, two particles are entangled when their physical states depend on one another's so that if we find one of them in state A, for example, the other will be in state B certainly [2]. However, until the physical state of a quantum particle is measured, it will be in a superposition of states, being neither in one nor the other until then [3], therefore, there is no way to say since the beginning of the entanglement, they were already decided on their properties.

Two entangled particles will only decide which state to assume when one is measured, then the other will automatically assume some state, regardless of the distance the particles are at that moment [4]. This fact makes us question how the other particle receives the information that its partner has been measured and now needs to assume a state consistent with that measurement?

\footnotetext{
* Correspondence: Ewaldo Ritzmann Street, number 822, Campo Lençol, Rio Negrinho, Santa Catarina, Brazil; e-mail: ritzmann.vinicius@gmail.com
}

Due to these facts, the article by Einstein et al raised the hypothesis that for there to be no need for instantaneous communication, the particles would have local hidden variables that would be adjusted at the beginning of the entanglement, and would dictate the behavior of the two particles from then on.

However, in 1964, Bell published an article [5] in which he demonstrated that if entanglement were the case of local hidden variables, the probability that certain measurements made in entangled pairs would occur would be different from what Quantum Mechanics predicted. Later, several experiments were carried out $[6,7]$ and demonstrated that the probability of these results occurring was following Quantum Mechanics, showing that the hypothesis of Einstein et al was wrong. Then much was discussed whether it would be possible to send information with entanglement.

It was discovered, as shown by Eberhard et al [8], that for many scenarios it is impossible to send information with entanglement. Although the states of the particles are related, they are still probabilistic.

However, what we show here is that not assuming anything about the speed of communication or even if there is communication between entangled particles, just using Quantum Mechanics as we currently have it, simple optical devices, and a protocol, there are ways to use entangled particles to send information. We will show that it is possible for two people, each with one of the entangled particles, to send information to each other instantly. 


\section{THE PROTOCOL}

Consider the following: in two places there are two people, A and B, who share an even number of entangled photon pairs. These photon pairs have entangled polarizations according to Equation (1) below:

$$
|\psi\rangle=\frac{1}{\sqrt{2}}\left(|V\rangle_{A}|V\rangle_{B}+|H\rangle_{A}|H\rangle_{B}\right)
$$

The states $|V\rangle$ and $|H\rangle$ correspond to orthogonal directions of polarization, so Equation (1) tells us that if we measure the polarization of the photons of one of these pairs, we will always find both polarized in the same direction $|V\rangle$ or $|H\rangle$ with a $50 \%$ chance of each result.

While they were together, A and B placed their photons in the same order, A's first photon is entangled with B's first, and so on. They also agreed on the following rules:

1. B will send information and A will only receive it;

2. When $\mathrm{A}$ arrives at his destination, he must place two photons at a time in the optical circuit of Figure 1 , he must place them in order, that is, the first with the second, the third with the fourth, and so on until there are no more photons;

3. Person A must always place as Photon A in the circuit the photon of the two that is in the lowest position of the order that $\mathrm{A}$ and $\mathrm{B}$ have agreed on. That is, when placing the first photon with the second in the circuit, the first must enter as Photon A and the second as Photon B. Another way of understanding this is odd photons are considered Photons A and even photons, Photons B;

4. After a fixed amount of photons crosses the circuit, an amount fixed by $\mathrm{A}$ and $\mathrm{B}$ as well, person $\mathrm{A}$ needs to check how many percent of the photons of that fixed group have been detected;

5. If A sees that $37.5 \%$ of the photons of a group were detected, A must understand this as the binary bit zero;

6. If A sees that $40.07 \%$ of the photons were detected, A must understand this as bit one;

The optical circuit of $\mathrm{A}$ is made of a polarizer always oriented at $90^{\circ}$ and a 50:50 non-polarizing beam splitter, considered here to be lossless; a beam splitter that allows half the photons to cross it while the other half is reflected. There is a detector at the output S5 and a barrier at the output $\mathrm{S} 4$ because we will not care about what goes out by $\mathrm{S} 4$.

Now we understand how person A must act to receive information, we will show how B can influence the results of A's circuit with the photons he has.

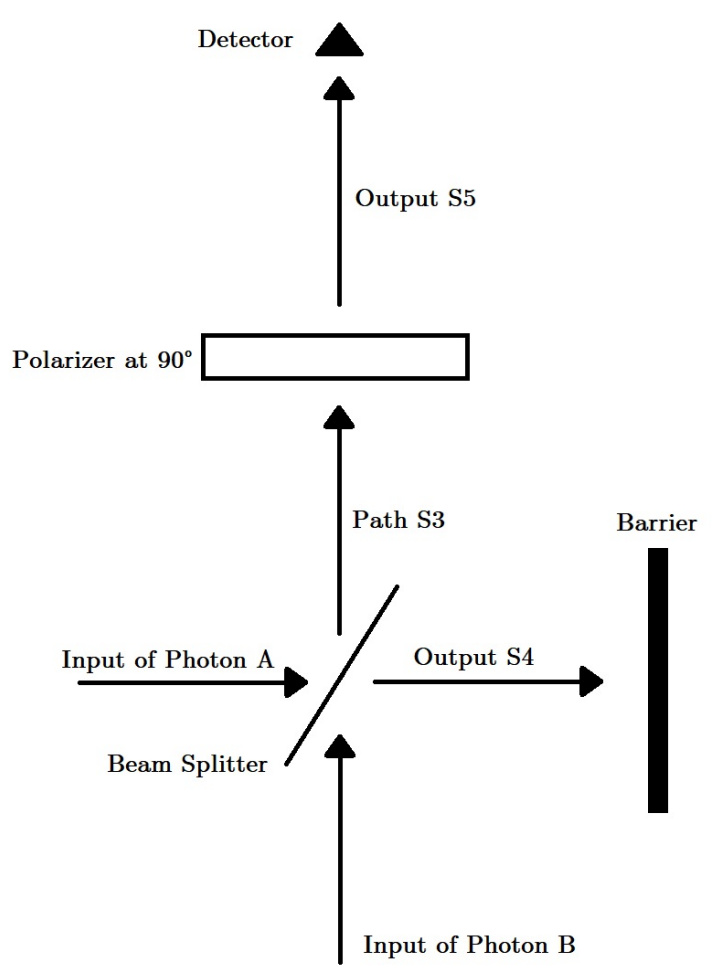

FIG. 1. OPTICAL CIRCUIT A. Optical circuit of A, made of a 50:50 beam splitter, a $90^{\circ}$ oriented polarizer, and a detector at the S5 output.

\section{SENDING INFORMATION}

Person B knows the fixed amount of photons A places in his circuit to receive a bit, he also knows which photons are within each of these groups, therefore, B also knows in which order A will receive the bits. Therefore, to send information, B needs to group his photons, each group containing the entangled photons with those photons in the groups of $\mathrm{A}$.

$\mathrm{B}$ also has an optical circuit. It is illustrated in Figure 2 .

The circuit of B has only two polarizers and barriers. Polarizer 1 must always be oriented at $90^{\circ}$ and polarizer 2 will be oriented at $90^{\circ}$ or $60^{\circ}$ depending on which information $\mathrm{B}$ wants to send to $\mathrm{A}$. We will soon explain this and do the math.

The rules for sending information are simple:

1. If $B$ wants $A$ to understand a group of photons contains bit zero, he must take the group of his photons that is entangled with the group of $\mathrm{A}$ and place them in his circuit, always respecting the preestablished order and the rule that odd photons enter as Photons $\mathrm{A}$ and even as Photons B, leaving polarizer 2 oriented at $90^{\circ}$ during the process, which must occur before the group in A crosses the circuit of $\mathrm{A}$; 

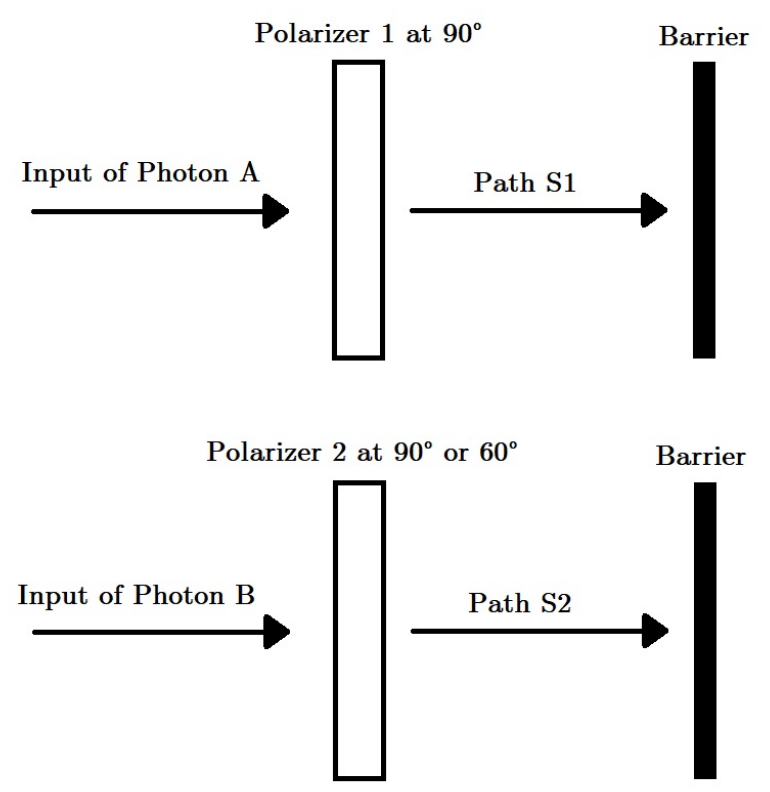

FIG. 2. OPTICAL CIRCUIT B. Optical circuit of B, made of two polarizers and barriers right after these polarizers because it does not matter any type of measurement made in these photons.

2. If $\mathrm{B}$ wants $\mathrm{A}$ to understand a group of photons contains bit one, he must do the same as he would do to send bit zero, however, leaving polarizer 2 oriented at $60^{\circ}$ during the process.

To understand how these actions lead to the behavior A expects to find in his circuit, we will mathematize all this.

First, if B wants to send bit zero, the two polarizers of his circuit will be oriented at $90^{\circ}$. The photons that enter as Photons $\mathrm{A}$ and $\mathrm{B}$ have the same polarization, given by Equation (1), the difference is that each is entangled with a different photon of $\mathrm{A}$.

The tensor product of the individual states of polarizations describes this situation where the photons enter the same circuit:

$$
\begin{aligned}
& \left|\psi^{\prime}\right\rangle=|\psi\rangle_{B \operatorname{In} A} \bigotimes|\psi\rangle_{B \operatorname{In} B}= \\
& \frac{1}{2}|V\rangle_{I n A}|V\rangle_{B I n A}|V\rangle_{I n B}|V\rangle_{B I n B}+ \\
& \frac{1}{2}|V\rangle_{\operatorname{In} A}|V\rangle_{B \operatorname{In} A}|H\rangle_{\operatorname{In} B}|H\rangle_{B \operatorname{In} B}+ \\
& \frac{1}{2}|H\rangle_{\operatorname{In} A}|H\rangle_{B \operatorname{In} A}|V\rangle_{\operatorname{In} B}|V\rangle_{B \operatorname{In} B}+ \\
& \frac{1}{2}|H\rangle_{\text {InA }}|H\rangle_{B \operatorname{In} A}|H\rangle_{\operatorname{In} B}|H\rangle_{B \operatorname{In} B}
\end{aligned}
$$

In Equation (2), we denoted as $B \operatorname{In} A$ the photon that enter as Photon $\mathrm{A}$ in the circuit and $B \operatorname{In} B$, the photon that enter as Photon B. The term InA refers to the photon that is entangled with photon $B \operatorname{In} A$ and $\operatorname{In} B$ refers to the one that is entangled with photon $B \operatorname{In} B$. Photons In $A$ and $\operatorname{In} B$ are photons of person $\mathrm{A}$.

According to Equation (2), the chance the two photons will cross the polarizers of the circuit simultaneously is $25 \%$ :

$$
\begin{aligned}
\left\langle V_{B \operatorname{In} A} V_{B \operatorname{In} B} \mid \psi^{\prime}\right\rangle & =\frac{1}{2}|V\rangle_{\operatorname{In} A}|V\rangle_{\operatorname{In} B} \\
\left\langle\psi^{\prime} \mid V_{B \operatorname{In} A} V_{B \operatorname{In} B}\right\rangle & =\frac{1}{2}\left\langleV | _ { \operatorname { I n } A } \left\langle\left. V\right|_{\operatorname{In} B}\right.\right.
\end{aligned}
$$

$$
\begin{array}{r}
\left\|\left\langle V_{B \operatorname{In} A} V_{B \operatorname{In} B} \mid \psi^{\prime}\right\rangle\right\|^{2}= \\
\left\langle\psi^{\prime} \mid V_{B \operatorname{In} A} V_{B \operatorname{In} B}\right\rangle\left\langle V_{B \operatorname{In} A} V_{B \operatorname{In} B} \mid \psi^{\prime}\right\rangle= \\
\frac{1}{2}\left\langle\left. V\right|_{\operatorname{In} A}\left\langle\left. V\right|_{\operatorname{In} B} \frac{1}{2} \mid V\right\rangle_{\operatorname{In} A} \mid V\right\rangle_{\operatorname{In} B}= \\
\frac{1}{4}=25 \%
\end{array}
$$

If the two photons cross the polarizers at the same time, both will be in state $|V\rangle$ before they reach the barriers. If only one of them crosses the polarizers, which is easy to see that will happen $50 \%$ of the time (we will not demonstrate this), we will have that one of the photons will be in the state $|V\rangle$ and the other, which did not cross the polarizer, should have been in the state $|H\rangle$.

If no photons cross the polarizers, what happens $25 \%$ of the time, both should have been in the state $|H\rangle$.

Everything that happens in B, happens before the photons entangled with those of $\mathrm{B}$ in $\mathrm{A}$ reach the circuit of A, this is stated by the protocol, so, when the photons of A arrive in his circuit, they are no longer entangled.

What happens is that $25 \%$ of the time what enters as Photon A and B in the beam splitter of A will be in the state $|V\rangle, 25 \%$ happens $|V\rangle$ and $|H\rangle, 25 \%$ happens $|H\rangle$ and $|V\rangle$, and $25 \%$ happens $|H\rangle$ and $|H\rangle$.

Each case is mathematized below. In these next equations, we used the mathematical formalism of the works of Wang [9] and $\mathrm{Ou}$ [10] concerning all the dynamics of the beam splitter of $\mathrm{A}$.

$$
\begin{array}{r}
\left|\psi_{B S}\right\rangle_{\text {output }, 1}=\frac{\sqrt{2}}{2}\left(|V\rangle_{1}|V\rangle_{1}-|V\rangle_{2}|V\rangle_{2}\right) \\
\left|\psi_{B S}\right\rangle_{\text {output }, 2}= \\
=\frac{1}{2}\left(|V\rangle_{1}|H\rangle_{2}-|V\rangle_{1}|H\rangle_{1}-|V\rangle_{2}|H\rangle_{2}+|V\rangle_{2}|H\rangle_{1}\right) \\
\frac{1}{2}\left(|H\rangle_{1}|V\rangle_{2}+|H\rangle_{1}|V\rangle_{1}+|H\rangle_{2}|V\rangle_{2}+|H\rangle_{2}|V\rangle_{1}\right)
\end{array}
$$




$$
\left|\psi_{B S}\right\rangle_{\text {output }, 4}=\frac{\sqrt{2}}{2}\left(-|H\rangle_{1}|H\rangle_{1}+|H\rangle_{2}|H\rangle_{2}\right)
$$

The full derivation of these results can be found in the appendix.

These four cases generate eight possible outputs for A's beam splitter, all with the same probability of occurring. Five of these outputs contain some photons that will leave the beam splitter by the path S3, which in the equations is denoted as output 1 , but not all of these photons leave the beam splitter in the correct polarization to cross the polarizer that exists on the path S3. Of the five outputs, only three contain some photons that leave by S3 path with the $|V\rangle$ polarization necessary to cross the polarizer as well.

In summary, what happens is that A will detect $37.5 \%$ of the photons. This is why we said that if A measured $37.5 \%$ of the photons, he should understand this as the zero binary because that is what happens when B places the photons of a group in his circuit with polarizer 2 oriented at $90^{\circ}$, what he would only do if he wanted to send bit zero.

The logic for sending bit one is the same as that for bit zero: person $\mathrm{B}$ will take the photons and place them in his circuit before the pairs of these photons cross the circuit of $\mathrm{A}$, but this time, polarizer 2 will be oriented at $60^{\circ}$.

Therefore, the mathematics of this case is very similar to the previous, the difference is since polarizer 2 is at $60^{\circ}$, the photons that try to cross it will either be polarized at $60^{\circ}$ after crossing, or they will collapse to $150^{\circ}$ if they do not cross.

We know that the photons entangled with them of $\mathrm{A}$ will also be in these orientations when they cross the circuit of $\mathrm{A}$, so it is enough we rewrite their polarizations as combinations of polarizations $|V\rangle$ and $|H\rangle$ to find what will come out of the beam splitter of $\mathrm{A}$.

If we do the math, and it is done in the appendix, we will see that as before, there will be eight possible outputs for the beam splitter, but this time, the outputs do not have all the same probability. Of the eight possible outputs, five have photons that exit by S3 path, and of these five, three have photons that cross the polarizer of S3 path.

What happens is A will detect $40.07(1428) \%$ of the photons. This is why, if A measures $40.07 \%$ of the photons, he must understand it as binary one, as this is what happens when B places a group in his circuit with polarizer 2 oriented at $60^{\circ}$, what he would only do if he wanted to send bit one.

Finally, as a comment, one can notice that if B rotates Polarizer 2 to other angles, the result of A's measurements will follow what says Equation (9) below:

$$
Q=\frac{100}{4}\left(1+\frac{\sin ^{2}(\theta)}{1+\sin ^{2}(\theta)}+\frac{\cos ^{2}(\theta)}{1+\cos ^{2}(\theta)}\right)
$$

This equation is obtained by doing the math with $\mathrm{B}$ using an undefined angle in polarizer 2 and it says that the percentage of photons A measures (Q) depends on the angle of polarizer 2. More information regarding the connection between the angle and the bases can be found in the appendix.

For example, within what we discussed earlier when B sends bit zero, he leaves polarizers 1 and 2 at the same angle, so the angle of Polarizer 2 is $90^{\circ}$, which results in $37.5 \%$ in Equation (9), exactly what we showed before. See that the result of Equation (9) also matches what we said in case the angle is $60^{\circ}$.

For this reason, it is possible to slightly increase the difference between the measurements of $\mathrm{A}$ of bits zero and one by using the angle $45^{\circ}$ (and not $60^{\circ}$ ) to send bit one. It is also possible to send information on a larger basis if $\mathrm{A}$ and $\mathrm{B}$ agree on more angles for the polarizer 2 and, whenever A measures the percentage corresponding to one of those angles, he will know which bit B is sending.

\section{CONCLUSION}

We showed person A monitoring only one detector can receive information from $B$ because part the time a percentage of photons is measured and part the time another percentage according to the will of person B.

Also, the information must travel faster than light, instantly, or person A could measure a percentage of photons that does not align with what person B did, and as soon as the information of what person $\mathrm{B}$ did reaches $\mathrm{A}$, the results would contradict each other. To avoid this kind of contradiction within Quantum Mechanics, the only way is for the measurement information to propagate instantly.

Thus, here we end the article with the proposal that someone carries out this experiment to show what happens in reality.

\section{Appendix: Full Derivations}

Here are the complete derivations of the outputs of the beam splitter of $\mathrm{A}$ when sending the bits zero and one.

Below, the derivation of the four possible outputs for the beam splitter in case of sending bit zero:

Case 1: 


$$
\begin{array}{r}
\left|\psi_{B S}\right\rangle_{\text {output }, 1}=\hat{U}\left|\psi_{B S}\right\rangle_{\text {input }, 1} \\
=\hat{U}|V\rangle_{1}|V\rangle_{2} \\
=\hat{U} \hat{a}_{1, V}^{\dagger} \hat{a}_{2, V}^{\dagger}|0\rangle \\
=\hat{U} \hat{a}_{1, V}^{\dagger} \hat{U}^{\dagger} \hat{U} \hat{a}_{2, V}^{\dagger} \hat{U}^{\dagger} \hat{U}|0\rangle \\
=\left(t \hat{b}_{1, V}^{\dagger}-r \hat{b}_{2, V}^{\dagger}\right)\left(t \hat{b}_{2, V}^{\dagger}+r \hat{b}_{1, V}^{\dagger}\right)|0\rangle \\
=\left(t^{2} \hat{b}_{1, V}^{\dagger} \hat{b}_{2, V}^{\dagger}+t r \hat{b}_{1, V}^{\dagger} \hat{b}_{1, V}^{\dagger}\right)|0\rangle+ \\
\left(-t r \hat{b}_{2, V}^{\dagger} \hat{b}_{2, V}^{\dagger}-r^{2} \hat{b}_{2, V}^{\dagger} \hat{b}_{1, V}^{\dagger}\right)|0\rangle \\
=\left(\left(t^{2}-r^{2}\right) \hat{b}_{1, V}^{\dagger} \hat{b}_{2, V}^{\dagger}\right)|0\rangle \\
+\left(\operatorname{tr}\left(\hat{b}_{1, V}^{\dagger} \hat{b}_{1, V}^{\dagger}-\hat{b}_{2, V}^{\dagger} \hat{b}_{2, V}^{\dagger}\right)\right)|0\rangle \\
=\frac{1}{2}\left(|V\rangle_{1}|V\rangle_{1}-|V\rangle_{2}|V\rangle_{2}\right)
\end{array}
$$

Normalized:

$$
\left|\psi_{B S}\right\rangle_{\text {output }, 1}=\frac{\sqrt{2}}{2}\left(|V\rangle_{1}|V\rangle_{1}-|V\rangle_{2}|V\rangle_{2}\right)
$$

Case 2:

$$
\begin{array}{r}
\left|\psi_{B S}\right\rangle_{\text {output }, 2}=\hat{U}\left|\psi_{B S}\right\rangle_{\text {input }, 2} \\
=\hat{U}|V\rangle_{1}|H\rangle_{2} \\
=\hat{U} \hat{a}_{1, V}^{\dagger} \hat{a}_{2, H}^{\dagger}|0\rangle \\
=\hat{U} \hat{a}_{1, V}^{\dagger} \hat{U}^{\dagger} \hat{U} \hat{a}_{2, H}^{\dagger} \hat{U}^{\dagger} \hat{U}|0\rangle \\
=\left(t \hat{b}_{1, V}^{\dagger}-r \hat{b}_{2, V}^{\dagger}\right)\left(t \hat{b}_{2, H}^{\dagger}-r \hat{b}_{1, H}^{\dagger}\right)|0\rangle \\
=\left(t^{2} \hat{b}_{1, V}^{\dagger} \hat{b}_{2, H}^{\dagger}-t r \hat{b}_{1, V}^{\dagger} \hat{b}_{1, H}^{\dagger}\right)|0\rangle+ \\
\left(-t r \hat{b}_{2, V}^{\dagger} \hat{b}_{2, H}^{\dagger}+r^{2} \hat{b}_{2, V}^{\dagger} \hat{b}_{1, H}^{\dagger}\right)|0\rangle
\end{array}
$$

Thus:

$$
\begin{array}{r}
\left|\psi_{B S}\right\rangle_{\text {output }, 2}= \\
=\frac{1}{2}\left(|V\rangle_{1}|H\rangle_{2}-|V\rangle_{1}|H\rangle_{1}-|V\rangle_{2}|H\rangle_{2}+|V\rangle_{2}|H\rangle_{1}\right)
\end{array}
$$

Case 3:

$$
\begin{array}{r}
\left|\psi_{B S}\right\rangle_{\text {output }, 3}=\hat{U}\left|\psi_{B S}\right\rangle_{\text {input }, 3} \\
=\hat{U}|H\rangle_{1}|V\rangle_{2} \\
=\hat{U} \hat{a}_{1, H}^{\dagger} \hat{a}_{2, V}^{\dagger}|0\rangle \\
=\hat{U} \hat{a}_{1, H}^{\dagger} \hat{U}^{\dagger} \hat{U} \hat{a}_{2, V}^{\dagger} \hat{U}^{\dagger} \hat{U}|0\rangle \\
=\left(t \hat{b}_{1, H}^{\dagger}+r \hat{b}_{2, H}^{\dagger}\right)\left(t \hat{b}_{2, V}^{\dagger}+r \hat{b}_{1, V}^{\dagger}\right)|0\rangle \\
=\left(t^{2} \hat{b}_{1, H}^{\dagger} \hat{b}_{2, V}^{\dagger}+t r \hat{b}_{1, H}^{\dagger} \hat{b}_{1, V}^{\dagger}\right)|0\rangle+ \\
\left(t r \hat{b}_{2, H}^{\dagger} \hat{b}_{2, V}^{\dagger}+r^{2} \hat{b}_{2, H}^{\dagger} \hat{b}_{1, V}^{\dagger}\right)|0\rangle
\end{array}
$$

Thus:

$$
\begin{array}{r}
\left|\psi_{B S}\right\rangle_{\text {output }, 3}= \\
\frac{1}{2}\left(|H\rangle_{1}|V\rangle_{2}+|H\rangle_{1}|V\rangle_{1}+|H\rangle_{2}|V\rangle_{2}+|H\rangle_{2}|V\rangle_{1}\right)
\end{array}
$$

Case 4:

$$
\begin{array}{r}
\left|\psi_{B S}\right\rangle_{\text {output }, 4}=\hat{U}\left|\psi_{B S}\right\rangle_{\text {input }, 4} \\
=\hat{U}|H\rangle_{1}|H\rangle_{2} \\
=\hat{U} \hat{a}_{1, H}^{\dagger} \hat{a}_{2, H}^{\dagger}|0\rangle \\
=\hat{U} \hat{a}_{1, H}^{\dagger} \hat{U}^{\dagger} \hat{U} \hat{a}_{2, H}^{\dagger} \hat{U}^{\dagger} \hat{U}|0\rangle \\
=\left(t \hat{b}_{1, H}^{\dagger}+r \hat{b}_{2, H}^{\dagger}\right)\left(t \hat{b}_{2, H}^{\dagger}-r \hat{b}_{1, H}^{\dagger}\right)|0\rangle \\
=\left(t^{2} \hat{b}_{1, H}^{\dagger} \hat{b}_{2, H}^{\dagger}-t r \hat{b}_{1, H}^{\dagger} \hat{b}_{1, H}^{\dagger}\right)|0\rangle+ \\
\left(t r \hat{b}_{2, H}^{\dagger} \hat{b}_{2, H}^{\dagger}-r^{2} \hat{b}_{2, H}^{\dagger} \hat{b}_{1, H}^{\dagger}\right)|0\rangle \\
=\left(\left(t^{2}-r^{2}\right) \hat{b}_{1, H}^{\dagger} \hat{b}_{2, H}^{\dagger}\right)|0\rangle \\
+\left(t r\left(-\hat{b}_{1, H}^{\dagger} \hat{b}_{1, H}^{\dagger}+\hat{b}_{2, H}^{\dagger} \hat{b}_{2, H}^{\dagger}\right)\right)|0\rangle \\
=\frac{1}{2}\left(-|H\rangle_{1}|H\rangle_{1}+|H\rangle_{2}|H\rangle_{2}\right)
\end{array}
$$

Normalized:

$$
\left|\psi_{B S}\right\rangle_{\text {output }, 4}=\frac{\sqrt{2}}{2}\left(-|H\rangle_{1}|H\rangle_{1}+|H\rangle_{2}|H\rangle_{2}\right)(\text { A }
$$

Below, the derivation of the four possible outputs for the beam splitter in case of sending bit one:

First, rewriting the polarization of the photons that enter as Photons B in the circuit of B on a basis where one of the axes is parallel to the polarizer's axis. This basis is represented in Figure 3 and is given by the transformation:

$$
\begin{aligned}
& |V\rangle=\sin \left(60^{\circ}\right)|R\rangle+\cos \left(60^{\circ}\right)|L\rangle \\
& |H\rangle=\cos \left(60^{\circ}\right)|R\rangle-\sin \left(60^{\circ}\right)|L\rangle
\end{aligned}
$$




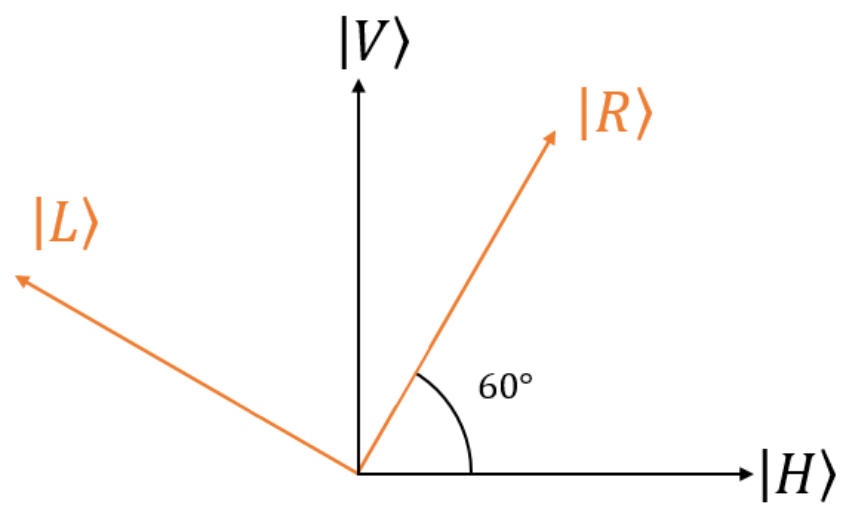

FIG. 3. THE BASES. Representation of the $|V\rangle$ and $|H\rangle$ polarization basis, where the entangled photon pairs were created, and $|R\rangle$ and $|L\rangle$ polarization basis which is the basis whose one axis is parallel to the orientation of polarizer 2 of B. The two bases are related by the $\theta$ angle.

Thus:

$$
\begin{aligned}
& |V\rangle=\frac{\sqrt{3}}{2}|R\rangle+\frac{1}{2}|L\rangle \\
& |H\rangle=\frac{1}{2}|R\rangle-\frac{\sqrt{3}}{2}|L\rangle
\end{aligned}
$$

The polarization of what enters as Photon B in the circuit is:

$$
\begin{array}{r}
|\psi\rangle_{B \operatorname{In} B}=\frac{1}{\sqrt{2}}\left(|V\rangle_{\operatorname{In} B} \mid\right. \\
\frac{1}{\sqrt{2}}\left(\frac{\sqrt{3}}{2}|R\rangle_{\operatorname{In} B}+\frac{1}{2}|L\rangle_{\operatorname{In} B}\right) \\
\left(\frac{\sqrt{3}}{2}|R\rangle_{B \operatorname{In} B}+\frac{1}{2}|L\rangle_{B \operatorname{In} B}\right)+ \\
\frac{1}{\sqrt{2}}\left(\frac{1}{2}|R\rangle_{\operatorname{In} B}-\frac{\sqrt{3}}{2}|L\rangle_{\operatorname{In} B}\right) \\
\left(\frac{1}{2}|R\rangle_{B \operatorname{In} B}-\frac{\sqrt{3}}{2}|L\rangle_{B \operatorname{In} B}\right)= \\
\frac{1}{\sqrt{2}}\left(|R\rangle_{\operatorname{In} B}|R\rangle_{B \operatorname{In} B}+|L\rangle_{\operatorname{InB}}|L\rangle_{B \operatorname{In} B}\right)
\end{array}
$$

In Equation A.11, the previous notation applies. The term BInB refers to the photon of $\mathrm{B}$ that enters as Photon $\mathrm{B}$ in the circuit of $\mathrm{B}$.

The state of the two photons that enter the circuit of $B$ is the tensor product of Equation A.11 and Equation 1, of the main text, for the case of it describing what enters as Photon A, we have:

$$
\begin{aligned}
& \left|\psi^{\prime}\right\rangle=|\psi\rangle_{B I n A} \bigotimes|\psi\rangle_{B I n B}= \\
& \frac{1}{2}|V\rangle_{\text {In } A}|V\rangle_{B \operatorname{In} A}|R\rangle_{\text {In } B}|R\rangle_{B \operatorname{In} B}+ \\
& \frac{1}{2}|V\rangle_{\text {InA }}|V\rangle_{B \operatorname{In} A}|L\rangle_{\text {InB }}|L\rangle_{B \operatorname{In} B}+ \\
& \frac{1}{2}|H\rangle_{\text {InA }}|H\rangle_{B \operatorname{In} A}|R\rangle_{\operatorname{In} B}|R\rangle_{B \operatorname{In} B}+ \\
& \frac{1}{2}|H\rangle_{\operatorname{In} A}|H\rangle_{B \operatorname{In} A}|L\rangle_{\operatorname{In} B}|L\rangle_{B \operatorname{In} B}
\end{aligned}
$$

For what enters as Photon A and B to cross the polarizers together, Photon A needs to be in state $|V\rangle$ and Photon B in state $|R\rangle$, this happens $25 \%$ of the time. There is a $50 \%$ chance only one of them will cross the polarizers. There is a $25 \%$ chance none will.

Thus, what arrives in A's circuit is already collapsed. $25 \%$ of the time happens to enter the circuit $|V\rangle$ and $|R\rangle$, $25 \%$ happens $|V\rangle$ and $|L\rangle, 25 \%$ happens $|H\rangle$ and $|R\rangle$ and $25 \%$ happens $|H\rangle$ and $|L\rangle$.

Knowing:

$$
\begin{aligned}
|R\rangle=\sin \left(60^{\circ}\right)|V\rangle & +\cos \left(60^{\circ}\right)|H\rangle= \\
= & \frac{\sqrt{3}}{2}|V\rangle+\frac{1}{2}|H\rangle \\
|L\rangle=\cos \left(60^{\circ}\right)|V\rangle & -\sin \left(60^{\circ}\right)|H\rangle= \\
= & \frac{1}{2}|V\rangle-\frac{\sqrt{3}}{2}|H\rangle
\end{aligned}
$$

The cases become:

Case 1: 


$$
\begin{array}{r}
\left|\psi_{B S}\right\rangle_{\text {output }, 1}=\hat{U}\left|\psi_{B S}\right\rangle_{\text {input }, 1} \\
=\hat{U}|V\rangle_{1}\left(\frac{\sqrt{3}}{2}|V\rangle_{2}+\frac{1}{2}|H\rangle_{2}\right) \\
=\hat{U}\left(\frac{\sqrt{3}}{2}|V\rangle_{1}|V\rangle_{2}+\frac{1}{2}|V\rangle_{1}|H\rangle_{2}\right) \\
=\hat{U}\left(\frac{\sqrt{3}}{2} \hat{a}_{1, V}^{\dagger} \hat{a}_{2, V}^{\dagger}+\frac{1}{2} \hat{a}_{1, V}^{\dagger} \hat{a}_{2, H}^{\dagger}\right)|0\rangle \\
=\frac{\sqrt{3}}{2} \hat{U} \hat{a}_{1, V}^{\dagger} \hat{U}^{\dagger} \hat{U} \hat{a}_{2, V}^{\dagger} \hat{U}^{\dagger} \hat{U}|0\rangle \\
+\frac{1}{2} \hat{U} \hat{a}_{1, V}^{\dagger} \hat{U}^{\dagger} \hat{U} \hat{a}_{2, H}^{\dagger} \hat{U}^{\dagger} \hat{U}|0\rangle \\
=\frac{\sqrt{3}}{2}\left(t \hat{b}_{1, V}^{\dagger}-r \hat{b}_{2, V}^{\dagger}\right)\left(t \hat{b}_{2, V}^{\dagger}+r \hat{b}_{1, V}^{\dagger}\right)|0\rangle \\
+\frac{1}{2}\left(t \hat{b}_{1, V}^{\dagger}-r \hat{b}_{2, V}^{\dagger}\right)\left(t \hat{b}_{2, H}^{\dagger}-r \hat{b}_{1, H}^{\dagger}\right)|0\rangle \\
=\frac{\sqrt{3}}{2}\left(t^{2} \hat{b}_{1, V}^{\dagger} \hat{b_{2, V}^{\dagger}}+t r \hat{b}_{1, V}^{\dagger} \hat{b}_{1, V}^{\dagger}\right)|0\rangle+ \\
\frac{\sqrt{3}}{2}\left(-t r \hat{b}_{2, V}^{\dagger} \hat{b}_{2, V}^{\dagger}-r^{2} \hat{b}_{2, V}^{\dagger} \hat{b}_{1, V}^{\dagger}\right)|0\rangle \\
+\frac{1}{2}\left(t^{2} \hat{b}_{1, V}^{\dagger} \hat{b}_{2, H}^{\dagger}-t r \hat{b}_{1, V}^{\dagger} \hat{b}_{1, H}^{\dagger}\right)|0\rangle+ \\
\frac{1}{2}\left(-t r \hat{b}_{2, V}^{\dagger} \hat{b}_{2, H}^{\dagger}+r^{2} \hat{b}_{2, V}^{\dagger} \hat{b}_{1, H}^{\dagger}\right)|0\rangle
\end{array}
$$

$$
\begin{array}{r}
\left|\psi_{B S}\right\rangle_{\text {output }, 2}=\hat{U}\left|\psi_{B S}\right\rangle_{\text {input }, 2} \\
=\hat{U}|V\rangle_{1}\left(\frac{1}{2}|V\rangle_{2}-\frac{\sqrt{3}}{2}|H\rangle_{2}\right) \\
=\hat{U}\left(\frac{1}{2}|V\rangle_{1}|V\rangle_{2}-\frac{\sqrt{3}}{2}|V\rangle_{1}|H\rangle_{2}\right) \\
=\hat{U}\left(\frac{1}{2} \hat{a}_{1, V}^{\dagger} \hat{a}_{2, V}^{\dagger}-\frac{\sqrt{3}}{2} \hat{a}_{1, V}^{\dagger} \hat{a}_{2, H}^{\dagger}\right)|0\rangle \\
=\frac{1}{2} \hat{U} \hat{a}_{1, V}^{\dagger} \hat{U}^{\dagger} \hat{U} \hat{a}_{2, V}^{\dagger} \hat{U}^{\dagger} \hat{U}|0\rangle \\
-\frac{\sqrt{3}}{2} \hat{U} \hat{a}_{1, V}^{\dagger} \hat{U}^{\dagger} \hat{U} \hat{a}_{2, H}^{\dagger} \hat{U}^{\dagger} \hat{U}|0\rangle \\
=\frac{1}{2}\left(t \hat{b}_{1, V}^{\dagger}-r \hat{b}_{2, V}^{\dagger}\right)\left(t \hat{b}_{2, V}^{\dagger}+r \hat{b}_{1, V}^{\dagger}\right)|0\rangle \\
-\frac{\sqrt{3}}{2}\left(t \hat{b}_{1, V}^{\dagger}-r \hat{b}_{2, V}^{\dagger}\right)\left(t \hat{b}_{2, H}^{\dagger}-r \hat{b}_{1, H}^{\dagger}\right)|0\rangle
\end{array}
$$

Normalized:

Normalized:

$$
\begin{array}{r}
\left|\psi_{B S}\right\rangle_{\text {output }, 3}=\hat{U}\left|\psi_{B S}\right\rangle_{\text {input }, 3} \\
=\hat{U}|H\rangle_{1}\left(\frac{\sqrt{3}}{2}|V\rangle_{2}+\frac{1}{2}|H\rangle_{2}\right) \\
=\hat{U}\left(\frac{\sqrt{3}}{2}|H\rangle_{1}|V\rangle_{2}+\frac{1}{2}|H\rangle_{1}|H\rangle_{2}\right) \\
=\hat{U}\left(\frac{\sqrt{3}}{2} \hat{a}_{1, H}^{\dagger} \hat{a}_{2, V}^{\dagger}+\frac{1}{2} \hat{a}_{1, H}^{\dagger} \hat{a}_{2, H}^{\dagger}\right)|0\rangle \\
=\frac{\sqrt{3}}{2} \hat{U} \hat{a}_{1, H}^{\dagger} \hat{U}^{\dagger} \hat{U} \hat{a}_{2, V}^{\dagger} \hat{U}^{\dagger} \hat{U}|0\rangle \\
+\frac{1}{2} \hat{U} \hat{a}_{1, H}^{\dagger} \hat{U}^{\dagger} \hat{U} \hat{a}_{2, H}^{\dagger} \hat{U}^{\dagger} \hat{U}|0\rangle \\
=\frac{\sqrt{3}}{2}\left(t \hat{b}_{1, H}^{\dagger}+r \hat{b}_{2, H}^{\dagger}\right)\left(t \hat{b}_{2, V}^{\dagger}+r \hat{b}_{1, V}^{\dagger}\right)|0\rangle \\
+\frac{1}{2}\left(t \hat{b}_{1, H}^{\dagger}+r \hat{b}_{2, H}^{\dagger}\right)\left(t \hat{b}_{2, H}^{\dagger}-r \hat{b}_{1, H}^{\dagger}\right)|0\rangle
\end{array}
$$

Case 3:$$
\left|\psi_{B S}\right\rangle_{\text {output }, 1}=
$$$$
\frac{\sqrt{24}}{4 \sqrt{5}}\left(|V\rangle_{1}|V\rangle_{1}-|V\rangle_{2}|V\rangle_{2}\right)
$$$$
+\frac{\sqrt{8}}{4 \sqrt{5}}\left(|V\rangle_{1}|H\rangle_{2}-|V\rangle_{1}|H\rangle_{1}-|V\rangle_{2}|H\rangle_{2}+|V\rangle_{2}|H\rangle_{1}\right)
$$

Case 2:

Normalized: 


$$
\begin{array}{r}
\left|\psi_{B S}\right\rangle_{\text {output }, 3}= \\
\frac{\sqrt{8}}{4 \sqrt{7}}\left(-|H\rangle_{1}|H\rangle_{1}+|H\rangle_{2}|H\rangle_{2}\right) \\
+\frac{\sqrt{24}}{4 \sqrt{7}}\left(|H\rangle_{1}|V\rangle_{2}+|H\rangle_{1}|V\rangle_{1}+|H\rangle_{2}|V\rangle_{2}+|H\rangle_{2}|V\rangle_{1}\right)
\end{array}
$$

$$
\begin{array}{r}
\left|\psi_{B S}\right\rangle_{\text {output }, 4}=\hat{U}\left|\psi_{B S}\right\rangle_{\text {input }, 4} \\
=\hat{U}|H\rangle_{1}\left(\frac{1}{2}|V\rangle_{2}-\frac{\sqrt{3}}{2}|H\rangle_{2}\right) \\
=\hat{U}\left(\frac{1}{2}|H\rangle_{1}|V\rangle_{2}-\frac{\sqrt{3}}{2}|H\rangle_{1}|H\rangle_{2}\right) \\
=\hat{U}\left(\frac{1}{2} \hat{a}_{1, H}^{\dagger} \hat{a}_{2, V}^{\dagger}-\frac{\sqrt{3}}{2} \hat{a}_{1, H}^{\dagger} \hat{a}_{2, H}^{\dagger}\right)|0\rangle \\
=\frac{1}{2} \hat{U} \hat{a}_{1, H}^{\dagger} \hat{U}^{\dagger} \hat{U} \hat{a}_{2, V}^{\dagger} \hat{U}^{\dagger} \hat{U}|0\rangle \\
-\frac{\sqrt{3}}{2} \hat{U} \hat{a}_{1, H}^{\dagger} \hat{U}^{\dagger} \hat{U} \hat{a}_{2, H}^{\dagger} \hat{U}^{\dagger} \hat{U}|0\rangle \\
=\frac{1}{2}\left(t \hat{b}_{1, H}^{\dagger}+r \hat{b}_{2, H}^{\dagger}\right)\left(t \hat{b}_{2, V}^{\dagger}+r \hat{b}_{1, V}^{\dagger}\right)|0\rangle \\
-\frac{\sqrt{3}}{2}\left(t \hat{b}_{1, H}^{\dagger}+r \hat{b}_{2, H}^{\dagger}\right)\left(t \hat{b}_{2, H}^{\dagger}-r \hat{b}_{1, H}^{\dagger}\right)|0\rangle
\end{array}
$$

Normalized:

$$
\begin{array}{r}
\left|\psi_{B S}\right\rangle_{\text {output }, 4}=\frac{\sqrt{24}}{4 \sqrt{5}}\left(|H\rangle_{1}|H\rangle_{1}-|H\rangle_{2}|H\rangle_{2}\right) \\
+\frac{\sqrt{8}}{4 \sqrt{5}}\left(|H\rangle_{1}|V\rangle_{2}+|H\rangle_{1}|V\rangle_{1}+|H\rangle_{2}|V\rangle_{2}+|H\rangle_{2}|V\rangle_{1}\right)
\end{array}
$$

The four cases generate eight possible outputs for the beam splitter of A, but the outputs do not have all the same probability. Of the eight possible outputs, five have photons that leave by S3 path and of these five outputs, only three have photons that cross the polarizer of S3 path.

What happens is A will detect 40.07(1428)\%.

Case 4:

Physics Physique Fizika 1, 195 (1964).

[6] Freedman, S. J. \& Clauser, J. F. Experimental test of local hidden-variable theories. Physical Review Letters 28, 938-941 (1972).

[7] Rauch, D. et al. Cosmic Bell Test Using Random Measurement Settings from High-Redshift Quasars. Physical Review Letters 121, 080403 (2018).

[8] Eberhard, P. H. \& Ross, R. R. Quantum field theory cannot provide faster-than-light communication. Foundations of Physics Letters 2, 127-149 (1989).

[9] Wang, K. Quantum Theory of Two-Photon Wavepacket Interference in a Beam Splitter. Preprint at https:// arxiv.org/abs/quant-ph/0311011 (2003).

[10] Ou, ZY. J. (2007) A Lossless Beam Splitter. In MultiPhoton Quantum Interference. Ed 1, Springer US, Boston, MA, pp 237-243. 
Figures
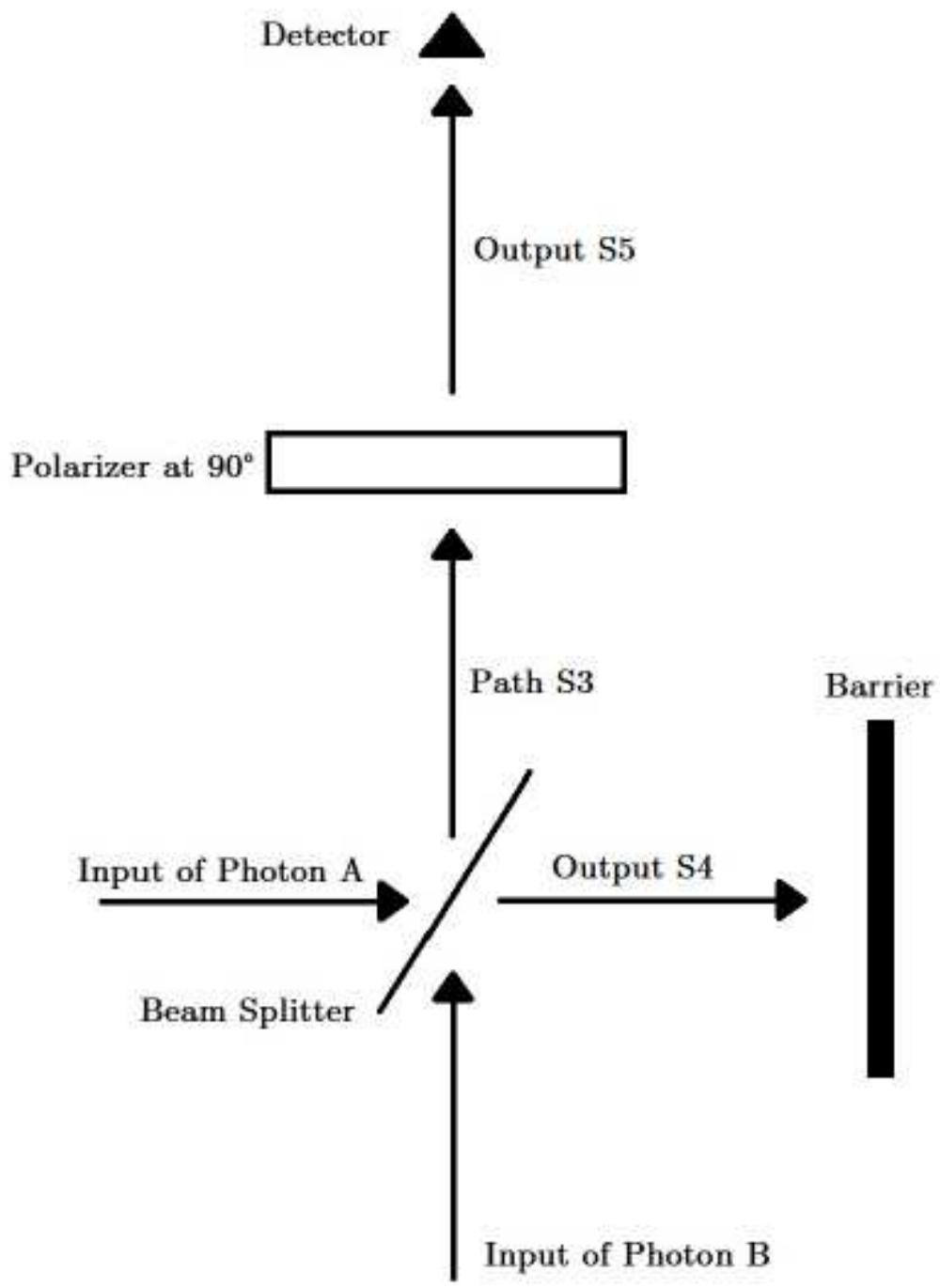

Figure 1

OPTICAL CIRCUIT A. Optical circuit of A, made of a 50:50 beam splitter, a 90冈degree oriented polarizer, and a detector at the S5 output. 

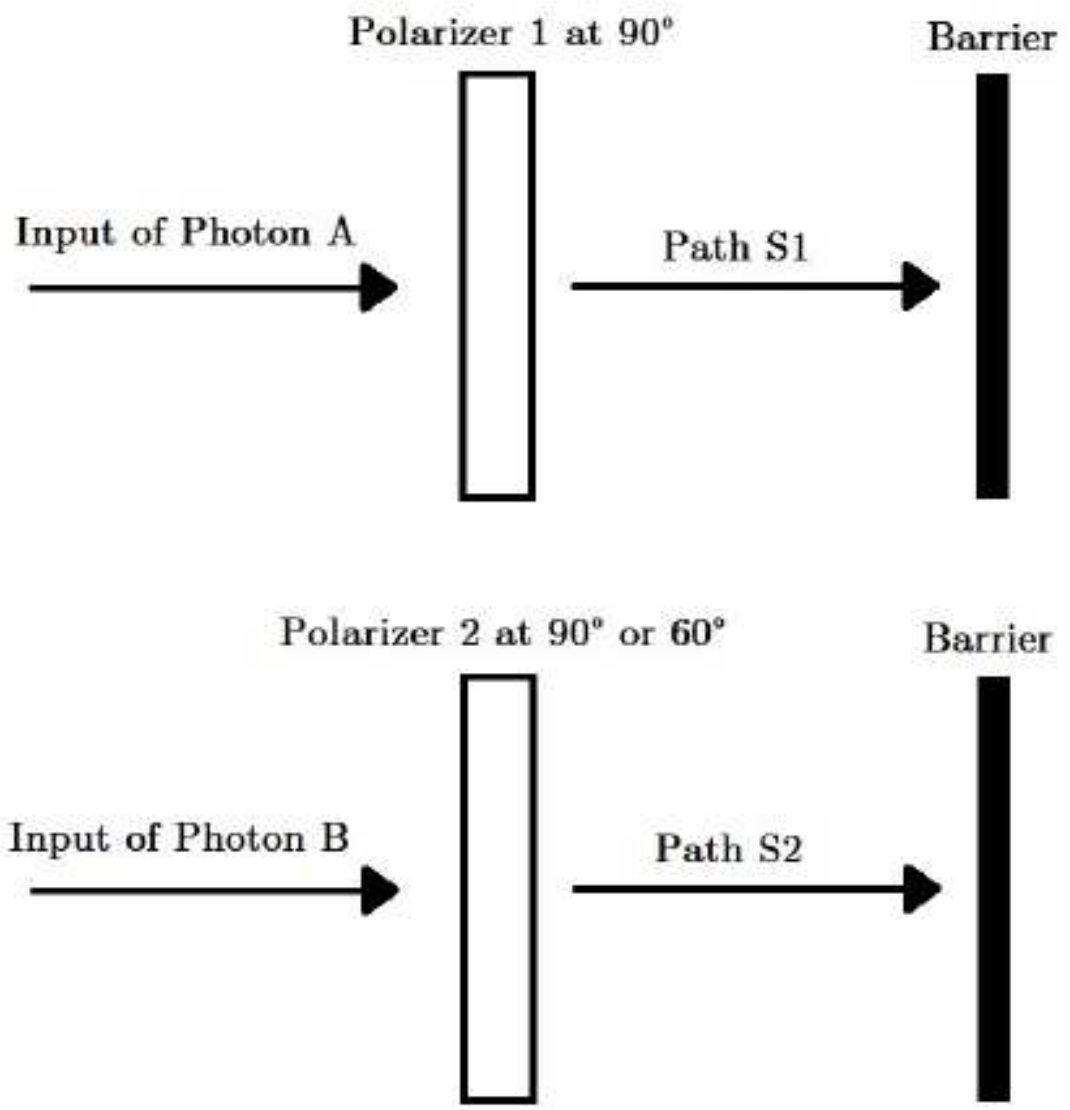

Figure 2

OPTICAL CIRCUIT B. Optical circuit of B, made of two polarizers and barriers right after these polarizers because it does not matter any type of measurement made in these photons. 


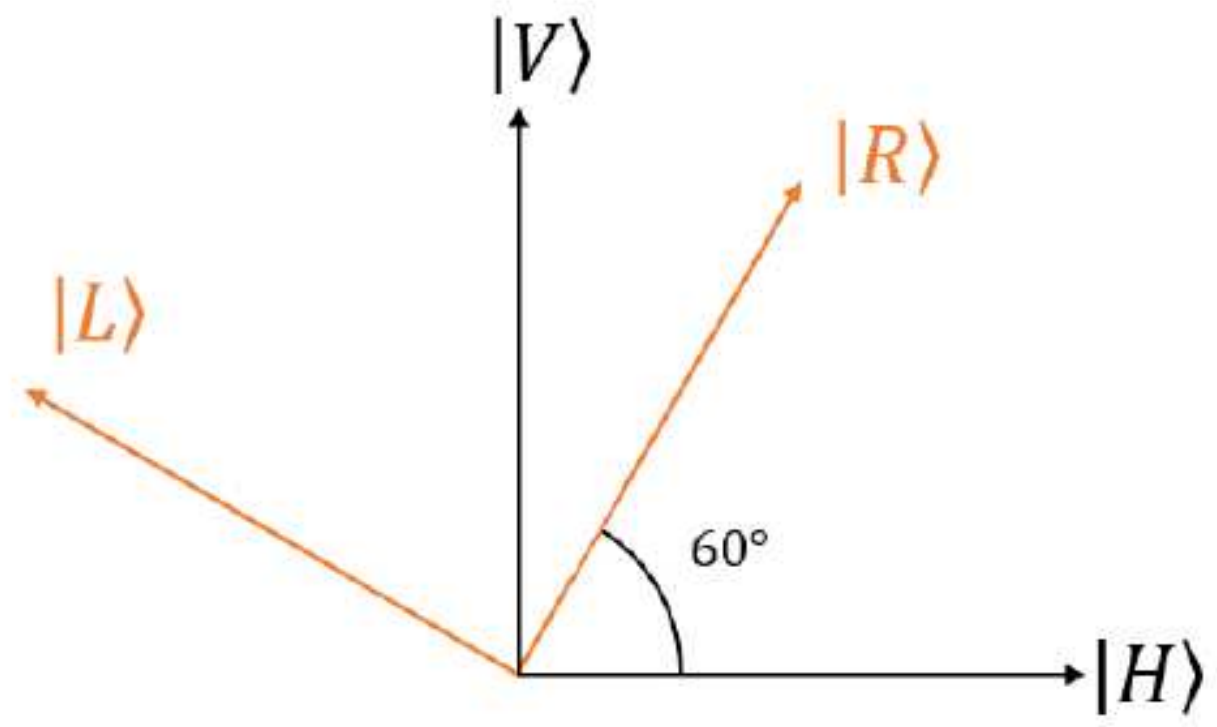

Figure 3

THE BASES. Representation of the $\mathrm{jV} i$ and $\mathrm{jHi}$ polarization basis, where the entangled photon pairs were created, and $\mathrm{jRi}$ and jLi polarization basis which is the basis whose one axis is parallel to the orientation of polarizer 2 of $B$. The two bases are related by the $\theta$ angle. 